\title{
How Group-Based Cardiovascular Health Education Affects Treatment Adherence and Blood Pressure Control among Insured Hypertensive Nigerians: A Pre-Test, Post-Test Study
}

\author{
Aina Olufemi Odusola ${ }^{1,2 *}$, Heleen Nelissen ${ }^{1}$, Marleen Hendriks ${ }^{1}$, Constance Schultsz ${ }^{1}$, \\ Ferdinand Wit ${ }^{1}$, Oladimeji Akeem Bolarinwa ${ }^{3}$, Tanimola Akande ${ }^{3}$, Charles Agyemang2, \\ Gbenga Ogedegbe ${ }^{4}$, Kayode Agbede5, Peju Adenusi', Akin Osibogun ${ }^{7}$, Karien Stronks², \\ Joke Haafkens $^{8}$ \\ ${ }^{1}$ Department of Global Health, Academic Medical Center, University of Amsterdam, Amsterdam Institute for \\ Global Health and Development, Amsterdam, Netherlands \\ ${ }^{2}$ Department of Public Health, Academic Medical Center, University of Amsterdam, Amsterdam, Netherlands \\ ${ }^{3}$ Department of Epidemiology and Community Health, University of Ilorin Teaching Hospital, Ilorin, Nigeria \\ ${ }^{4}$ Division of Health and Behavior, Center for Healthful Behavior Change, Department of Population Health, NYU \\ School of Medicine, New York, USA \\ ${ }^{5}$ Ogo Oluwa Hospital, Bacita, Nigeria \\ ${ }^{6}$ Hygeia Community Health Care, Hygeia HMO, Lagos, Nigeria \\ ${ }^{7}$ Department of Community Health, Lagos University Teaching Hospital, Lagos, Nigeria \\ ${ }^{8}$ Department of General Practice, Academic Medical Center, University of Amsterdam, Amsterdam, Netherlands \\ Email: *A.O.Odusola@amc.uva.nl, h.nelissen@aighd.org, m.hendriks@aighd.org, schultsz@gmail.com, \\ f.wit@aighd.org, drdeji@yahoo.com, akandetm@yahoo.com, c.o.agyemang@amc.uva.nl, Olug- \\ benga.ogedegbe@nyumc.org, agbedejk@yahoo.com, dradenusi@hygeiahmo.com, \\ akinosibogun@yahoo.co.uk, k.stronks@amc.uva.nl, j.a.haafkens@amc.uva.nl
}

Received 13 June 2015; accepted 27 July 2015; published 30 July 2015

Copyright (C) 2015 by authors and Scientific Research Publishing Inc.

This work is licensed under the Creative Commons Attribution-NonCommercial International License (CC BY-NC).

http://creativecommons.org/licenses/by-nc/4.0/

(c) (i) (8) Open Access

\section{Abstract}

In sub Saharan Africa (SSA), access to affordable hypertension care through health insurance is increasing. But due to poor adherence, hypertension treatment outcomes often remain poor. Patient-centered educational interventions may reverse this trend. Using a pre-test/post-test design,

${ }^{*}$ Corresponding author.

How to cite this paper: Odusola, A.O., Nelissen, H., Hendriks, M., et al. (2015) How Group-Based Cardiovascular Health Education Affects Treatment Adherence and Blood Pressure Control among Insured Hypertensive Nigerians: A Pre-Test, Post-Test Study. World Journal of Cardiovascular Diseases, 5, 181-198. http://dx.doi.org/10.4236/wjcd.2015.57021 
in this study we investigated the effects of a structured cardiovascular health education program (CHEP) on treatment adherence, blood pressure (BP) control and body mass index (BMI) among Nigerian hypertensive patients who received guideline-based care in a rural primary care facility, in the context of a community based health insurance program. Study participants included 149 insured patients with uncontrolled BP and/or poor self-reported medication adherence after 12 months of guideline-based care. All patients received three group-based educational sessions and usual primary care over 6 months. We evaluated changes in self-reported adherence to prescribed medications and behavioral advice (primary outcomes); systolic BP (SBP) and/or diastolic BP (DBP) and BMI (secondary outcomes); and beliefs about hypertension and medications (exploratory outcomes). Outcomes were analyzed with descriptive statistics and regression analysis. 140 patients completed the study (94\%). At 6 months, more participants reported high adherence to medications and behavioral advice than at baseline: respectively, $101(72 \%)$ versus $70(50 \%)$, (p $<0.001)$ and $126(90 \%)$ versus $106(76 \%),(p<0.001)$. Participants with controlled BP doubled from $34(24 \%)$ to $65(46 \%),(p=0.001)$. The median SBP and DBP decreased from 129.0 to 122.0 $\mathrm{mmHg},(\mathrm{p}=0.002)$ and from 80.0 to $73.5 \mathrm{mmHg},(\mathrm{p}<0.001)$, respectively. BMI did not change $(\mathrm{p}=$ 0.444). Improved medication adherence was associated with a decrease in medication concerns ( $p$ $=0.045)$ and improved medication self-efficacy $(p<0.001)$. By positively influencing patient perceptions of medications, CHEP strengthened medication adherence and, consequently, BP reduction among insured hypertensive Nigerians. This educational approach can support cardiovascular disease prevention programs for Africa's growing hypertensive population.

\section{Keywords}

BP-Control, Education, Adherence, Self-Efficacy, Insurance, Nigeria

\section{Introduction}

Hypertension is a major risk factor for cardiovascular disease (CVD) [1]. The highest prevalence of adults with hypertension has been found in SSA, where a quarter of all premature deaths are due to this condition [1] [2]. In Nigeria, 49\% of the adults, aged 25 years and older, had hypertension in 2008 [2]. Long-term treatment with behavioral interventions alone, or in combination with drugs can lower BP and, thereby, the risk of developing CVD [1] [3]-[5]. However, as in many other African countries, in Nigeria anti-hypertensive treatment coverage is low and treatment outcomes are poor [6] [7].

Previous research in Nigeria and other countries in SSA has indicated that health system interventions such as health insurance coverage, organizational support to facilities and the training of health professionals can facilitate the access to and delivery of high quality CVD prevention care in primary care settings [8]-[14]. But even if high quality care is available, there is evidence that many patients fail to adhere to the recommended treatment and do not meet their treatment goals [15]-[17]. For that reason, the World Health Organization has emphasized that any attempt to improve hypertension and cardiovascular care should also address barriers to treatment adherence [18].

Theoretical models of health behavior have proposed that patients and health professionals have different explanatory models or beliefs about health and illness and that beliefs held by patients are important determinants of treatment adherence [19] [20]. This has been confirmed by empirical studies of treatment adherence among patients with hypertension [20] [21]. Evidence suggests that patient education can be effective in supporting treatment adherence and hypertension self-management, especially if the educational programs pay attention to underlying barriers to adherence such as patients' beliefs and concerns about the nature of hypertension and the prescribed treatment, medication self-efficacy and specific social, cultural and individual barriers to optimal hypertension management [22]-[25]. A systematic review of studies on various strategies for improving the quality of primary hypertension care in high income countries concludes that organizational health system changes and patient education have the biggest impact on blood pressure (BP) outcomes [26]. Some recent primary care studies from Nigeria also demonstrate that organizational quality improvement interventions can lead to improved clinical outcomes in hypertensive patients [14] [27] [28]. However, to date little information is 
available about the impact of patient education on hypertension management in low resource communities in Nigeria or other countries in SSA. We had the opportunity to investigate this issue in a rural primary care facility in Kwara State Nigeria, where clinical guidelines were introduced in 2010 in order to improve the quality of hypertension management for patients enrolled in a Community Based Health Insurance (CBHI) program. To strengthen this quality improvement program, we implemented a tailored community-based cardiovascular health education program (CHEP) for patients. CHEP was developed and structured on the basis of the theoretical frameworks of Kleinman and Leventhal [19] [20], and the content was based on findings from a previous qualitative study on perspectives on hypertension and treatment adherence among hypertensive patients from the local community [29]. Because patients in this facility had been offered free guideline-based cardiovascular prevention care, in the context of their health insurance, the setting allowed for a focused analysis of the value of patient education. The aims of this study were to evaluate the effects of CHEP on treatment adherence, BP control and body mass index (BMI) and to explore to what extent the changes that occurred in medication adherence after patients had completed CHEP were related to changes in the underlying determinants of adherence behavior that were addressed during the training (i.e. patients' perceptions of hypertension, medication, and selfefficacy).

\section{Methods}

The study design has been published previously [30]. This section summarizes the main procedures.

\subsection{Study Site and Context}

The study was conducted at Ogo Oluwa hospital (OOH) in Bacita, a rural low-income community in Kwara State. $\mathrm{OOH}$ was contracted by the Kwara State Health Insurance (KSHI) program to provide primary and limited secondary care to patients who are enrolled in the health insurance plan. Financed by an international organization-the Health Insurance Fund (HIF) [31], the KSHI program was launched in Kwara State in 2007 with the aim to provide subsidized health insurance for low and middle income groups.

As part of the insurance company's quality assurance program, $\mathrm{OOH}$ had implemented guidelines and treatment protocols for CVD prevention [4] [32] [33], and upgraded its diagnostic equipment and medical record system. In a previously reported study, Quality Improvement Cardiovascular care Kwara (QUICK)-I, we evaluated the feasibility and quality of this program [28] [34]. The QUICK-I study included 349 insured patients with hypertension and/or diabetes from OOH between June 2010 and January 2011, followed these patients over a period of 12 months and assessed them for health-related and other outcomes, including medication adherence, systolic BP (SBP) and diastolic BP (DBP). Of the 349 included patients 323 completed the study at one year of follow up [28].

\subsection{Study Design and Participants}

The present study, QUICK-II, was an observational one-group pre-test post-test study of 149 patients who had completed the QUICK-I study [30]. QUICK-I patients were recruited for QUICK-II if the following criteria were present at the one-year follow up assessment of QUICK-I: enrolled in KSHI program; registered at OOH; aged $\geq$ 18 years; diagnosed with hypertension; having uncontrolled $\mathrm{BP}$ (SBP $\geq 140 \mathrm{mmHg}$ or DBP $\geq 90 \mathrm{mmHg}$ without co-morbidity—diabetes, renal disease, cardiovascular diseases—or SBP $\geq 130 \mathrm{mmHg}$ or DBP $\geq 80 \mathrm{mmHg}$ with co-morbidity) and/or being non-adherent to behavioral recommendations or prescribed medications (score $<8$ on the Morisky Medication Adherence Scale (MMAS-8) [35] [36]; and willing to provide informed consent. Pregnant or lactating females were excluded from the study.

Baseline assessments for QUICK-II patients $\left(\mathrm{T}_{0}\right)$ were conducted between November 2011 and March 2012, and six-month-follow up assessments $\left(T_{1}\right)$ were conducted between April 2012 and September 2012. Between $\mathrm{T}_{0}$ and $\mathrm{T}_{1}$, all included patients were offered cardiovascular health education program (CHEP) counseling, in addition to their regular hypertension care. Two trained research nurses, fluent in local languages, conducted baseline and follow-up assessments of physiological and self-report measures. Questionnaires for assessing selfreport measures were designed and piloted in English, and translated into the two dominant local languages (Yoruba and Nupe). Patients received a reminder 2 days before the scheduled study visits. Incurred travel costs were reimbursed if study visits took place outside a patient's usual clinic days. 


\subsection{Intervention-CHEP}

The intervention, CHEP, consisted of: (i) three group-based educational sessions; and (ii) culturally tailored written and audio-visual educational materials (see table, Additional File 1). The content of CHEP was inspired by a hypertension education program that was developed by Beune et al. [23], and results of a previous interview study with hypertensive patients of $\mathrm{OOH}$ [29]. All patients were randomly assigned to a group of 12 - 15 "trainees" which held the same composition throughout the program. The CHEP training sessions took place at $\mathrm{OOH}$, at respectively 2, 6 and 14 weeks after $\mathrm{T}_{0}$. The first session lasted 2 hours and the second and third sessions lasted 2.5 hours each. The training was given by the researcher (AOO) and a trained research nurse. Sessions were held in the languages of choice of the group and interactive training techniques were used in all sessions.

\subsection{Outcome Measures and Data Collection}

\subsubsection{Primary and Secondary Outcomes}

The primary outcomes were the proportion of study participants who had improved self-reported adherence to medication and behavioral recommendations at six months past baseline.

Medication adherence was assessed with the MMAS-8 [35] [36]. The MMAS-8 asks participants 7 "yes" or "no" questions and 1 question that can be answered on a 5-point Likert scale. Low adherence is defined as MMAS-8 scores < 6; medium adherence as scores ranging from 6-to $<8$, and high adherence as a score of 8 [36]. We defined improvement in medication adherence as a shift to a higher category of adherence between $\mathrm{T}_{0}$ and $\mathrm{T}_{1}$ (e.g. from low to medium adherence) or as 'high adherence' (MMAS-8 score of 8) at both points in time.

Adherence to behavioral advice was measured with the question "to what extent do you follow the behavioral advice from your doctor about smoking/nutrition/drinking alcohol/losing weight/physical activity or something else"? Answers were provided on a 4-point Likert scale, (1) never, (2) sometimes, (3) usually or (4) always. We defined improvement in adherence to behavioral recommendations as a shift from lower to a higher category of adherence between $T_{0}$ and $T_{1}$ or as high adherence (category 4) at both points in time.

Secondary outcomes were: the proportion of patients who showed an improvement in BP between $T_{0}$ and $T_{1}$; and the proportion of patients who showed a decrease in body mass index (BMI) by $\geq 1 \mathrm{unit} \mathrm{kg} / \mathrm{m}^{2}$ between $\mathrm{T}_{0}$ and $\mathrm{T}_{1}$.

BP was measured three times, 5 minutes apart, with an automated BP monitor (Omron M6 Comfort, OMRON Corporation, Kyoto, Japan), after the patient had been seated for 5 minutes. The second and third readings were averaged to calculate the SBP and DBP. A controlled BP was defined as a SBP of $<140 \mathrm{mmHg}$ and a DBP of $<90$ $\mathrm{mmHg}$ for patients without co-morbidity, or as SBP $<130 \mathrm{mmHg}$ and DBP $<80 \mathrm{mmHg}$ for patients with comorbidity. Improvement in BP was defined as $\mathrm{a} \geq 10 \%$ decrease in SBP and/or DBP between $\mathrm{T}_{0}$ and $\mathrm{T}_{1}$ or having a controlled BP at both $\mathrm{T}_{0}$ and $\mathrm{T}_{1}$.

BMI was calculated from measures of the patient's height and weight. Weight was measured with validated Omron BF 400 weighing scale, and height with a validated Leicester Stadiometer SECA 217. Both measures were taken without the patient wearing shoes and/or heavy clothing. Measurements were recorded to the nearest $0.1 \mathrm{~cm}$ (height) and $0.1 \mathrm{~kg}$ (weight). Normal weight was defined as a BMI below $25 \mathrm{~kg} / \mathrm{m}^{2}$, overweight as a BMI between 25 and $29.9 \mathrm{~kg} / \mathrm{m}^{2}$, and obesity as a BMI $\geq 30 \mathrm{~kg} / \mathrm{m}^{2}$. Improvement in BMI was defined as a $\geq 1$ unit $\mathrm{kg} / \mathrm{m}^{2}$ decrease in BMI between $\mathrm{T}_{0}$ and $\mathrm{T}_{1}$ for patients with a baseline BMI $\geq 25 \mathrm{~kg} / \mathrm{m}^{2}$.

\subsubsection{Other Measures}

The intervention targeted some determinants of medication adherence: patients' perceptions of hypertension, medication, and medication self-efficacy. We measured these variables in order to obtain a better understanding of the expected change in primary outcomes.

Patients' perceptions of hypertension were assessed with the well validated Revised Illness Perception Questionnaire (IPQ-R) [37]. The IPQ-R asks participants to provide answers to statements on a 5-point Likert scale ( 1 = "strongly disagree", 5 = "strongly agree") on 9 dimensions of illness (hypertension), 7 of which were used in this study. Scores are totaled and overall score represents the degree to which hypertension is perceived as threatening or benign. High scores on the dimensions 1) emotional representations, 2) timeline chronic, 3) consequences, 4) timeline cyclical represent strongly held beliefs about 1) number of symptoms attributed to hypertension, 2) its chronicity, 3) its negative consequences and 4) its cyclical nature. High scores on the personal 
control, treatment control and illness coherence dimensions represent positive beliefs about the controllability of hypertension and a personal understanding of the illness.

Patients' beliefs about medicines were assessed with the Beliefs about Medicines Questionnaire (BMQ) [38]. BMQ is a well validated 18-item tool that consists of two sections. In this study we used only section 1 (BMQspecific) which consists of two 5-item subscales. The first scale (Specific-necessity) assesses hypertensive patients' beliefs about how necessary it is to take medications in order to improve/maintain their health. The second scale (Specific-concern) assesses respondents" "concerns" about potential adverse consequences from taking their medications. BMQ uses 5-point Likert questions ranging from $1=$ "strongly disagree" to $5=$ "strongly agree". The respondents' scores on each item are totaled. Higher scores indicate stronger beliefs about the necessity of taking medicines and concerns about adverse effects of medications.

Medication self-efficacy was measured with the shortened Medication Adherence Self-Efficacy Scale (MASES-R) [39] [40]. This 13-item scale assesses the patients' beliefs in their confidence to adhere to prescribed anti-hypertensive medications under a variety of challenging situations, such as when busy at home, when there are symptoms, while traveling etc. Items are scored using a 4-point Likert scale, $(1=$ "not at all sure", 4 = "extremely sure"). The scores on all items are totaled. Higher scores indicate higher self-reported medication adherence self-efficacy.

In order to collect additional information about self-reported health behaviors, participants were asked questions about physical activity, dietary salt intake, alcohol and tobacco use. Daily moderate physical activity was defined as performing sports or exercise (e.g. walking to the market, performing heavy work) in addition to one's normal daily activities such as dressing, washing and walking. Salt use was defined as adding any salt (a little/a lot) when cooking or when eating food. Alcohol use was defined as any self-reported use of alcohol daily, weekly or monthly. Tobacco use is self-reported use of any tobacco products, such as cigarettes, cigars or pipes. Information about patients’ socio-demographic characteristics was obtained from the QUICK-I study.

\subsection{Statistical Analysis}

Data were analyzed using STATA, version 12.0 (StataCorp LP, College Station, Texas, USA). Adherence to medications and behavioral advice, BP control and BMI were calculated using descriptive statistics. Changes between $T_{0}$ and $T_{1}$ were compared using the Wilcoxon signed rank test for categorical and continuous variables and the McNemar exact test for binary variables. Changes in illness perceptions, medication beliefs and self-efficacy between $\mathrm{T}_{0}$ and $\mathrm{T}_{1}$ were compared using the Wilcoxon signed rank test.

A first multivariable logistic regression analysis was performed to evaluate the association between improvement in BP between $\mathrm{T}_{0}$ and $\mathrm{T}_{1}$ (secondary outcome) and medication and behavioral adherence (primary outcome).

A second multivariable logistic regression analysis was performed to explore the associations between improvement in adherence to medications between $\mathrm{T}_{0}$ and $\mathrm{T}_{1}$ (primary outcome) and illness perceptions, medication beliefs, and self-efficacy. The illness perceptions, medication beliefs and self-efficacy variables showing a p-value below 0.2 in a univariate analysis were included in the multivariate model.

In both models no control variables (i.e. age, gender, level of education, ethnicity, co-morbidities, etc.) were included since no substantial change was expected in these variables during the study period. The odds ratios (OR), 95\% confidence interval (CI) and p-values were reported.

To evaluate the association between change in SBP and DBP (between $\mathrm{T}_{0}$ and $\mathrm{T}_{1}$ ) and medication and behavioral adherence, two multivariable linear regression analyses were performed. Coefficients (in mmHg), 95\% CI and p-values were reported and similar to the logistic regression models, no control variables were included in the analyses.

Endline measurements in QUICK-I were used to determine patients eligibility for QUICK-II (uncontrolled BP and/or non-adherence to medications). On average, there was a gap of 4.7 months between the endline QUICK-I assessment and the baseline QUICK-II assessment $\left(T_{0}\right)$. During this period, 33\% $(n=49)$ of the eligible QUICK-II patients had improved in BP and/or medication adherence (see Additional file 2). The number of patients with a controlled BP at $\mathrm{T}_{0}$ was high (59.7\%), resulting in a low power for the first multivariable regression analysis. In the original design of the study [30], our definitions of the secondary outcome measure, BP improvement was very strict $\left(\mathrm{a} \geq 10 \%\right.$ decrease in SBP and/or DBP between $\mathrm{T}_{0}$ and $\mathrm{T}_{1}$ or having a controlled BP at both $\mathrm{T}_{0}$ and $\mathrm{T}_{1}$ ). We believe, however, that any BP decrease can be favorable in the studied participants. For this 
reason, the research group decided to measure the secondary outcome also by assessing the BP improvement continuously as the delta of SBP and DBP between $\mathrm{T}_{0}$ and $\mathrm{T}_{1}$ and to perform the two multivariable linear regression analyses mentioned above.

\subsection{Ethics}

Ethical approval for the study was obtained on 30th March, 2010 from the Ethics committee of the University of Ilorin Teaching Hospital, Kwara State (Ref: UITH/CAT/189/13/13). Patients were adequately informed about the study and informed consents were taken prior to commencement of study by signature or fingerprint.

\section{Results}

\subsection{Patient Characteristics}

The QUICK-II cohort consisted of 149 patients. Participant flow is shown in Figure 1.

Out of 323 patients who completed QUICK-I study 156 were referred to QUICK-II, 7 of whom were excluded because they had diabetes but not hypertension. The remaining 149 patients were included in QUICK-II. For different reasons, nine patients (6\%) were lost to follow-up. Of those who completed the study $(\mathrm{n}=140)$, 132 (94\%) attended all three CHEP sessions.

Table 1 shows participants' socio-demographic characteristics. The median age was 56.5 years (IQR: 49.4 65.5), 63 (42\%) were males and 85 (57\%) were not formally educated.

\subsection{Changes in Adherence to Medication and Behavioral Recommendations, BP and BMI}

As Table 2 shows, medication adherence improved during the study period $(\mathrm{p}<0.001)$. As compared to $\mathrm{T}_{0}$, more patients reported a high level of medication adherence (MMAS-8 $=8$ ) at $\mathrm{T}_{1}: \mathrm{N}=70(50 \%)$ versus $\mathrm{N}=101$ (72\%). A similar pattern was observed for adherence to behavioral recommendations; the proportion of patients

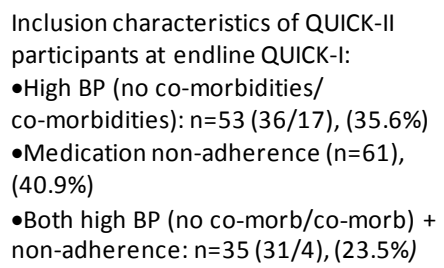

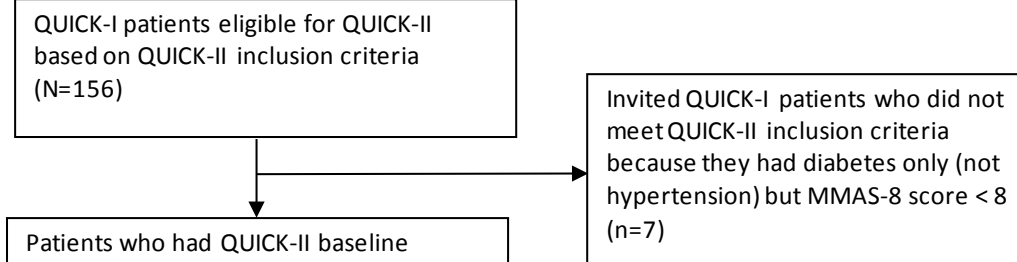

Invited QUICK-I patients who did not meet QUICK-II inclusion criteria because they had diabetes only (not hypertension) but MMAS- 8 score $<8$ $(n=7)$

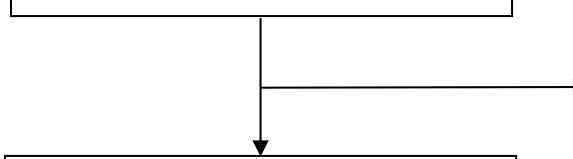

Patients who participated in CHEP sessions ( $n=140)$ :

-Attended 3 CHEP sessions $(n=132)$

-Attended at least 1 CHEP session $(n=6)$

-Missed all 3 CHEP sessions $(n=2)$
QUICK-II participants lost to follow-up during CHEP, $n=9$ (6\%):

-Died $(n=1)$

-Relocated $(n=4)$

-Discontinued $(n=1)$

-Unknown reason $(n=3)$ 
Table 1. Characteristics of study participants $(\mathrm{N}=149)$.

\section{Characteristics}

Male, n (\%)

Age in years, median (IQR)

Educational level attained, n (\%)

No school at all

$<$ Primary

Primary

Secondary

Tertiary

\section{Employment status, n (\%)}

Farmer/fisherman

\section{N (\%)}

63 (42.3)

56.5 (49.4 - 65.5)

85 (57.0)

3 (2.0)

33 (22.1)

12 (8.1)

16 (10.7)

36 (24.2)

59 (39.6)

23 (15.4)

31 (20.8)

Other

133 (89.3)

Married

16 (10.7)

Religion, n (\%)

Muslim

Christian

69 (46.3)

Ethnicity, n (\%)

Nupe

Yoruba

67 (45.0)

Igbo

$10(6.7)$

Other

12 (8.1)

Household size, median (IQR)*

Co - morbidities**, n (\%)

None

Diabetes

CVD

Renal disease

4 (2.7)

Two or three co - morbidities

$<1.5$ year $100(67.1)$

$>1.5$ year

Number of prescribed antihypertensive pills per day**, n (\%)***

$<=4$ pills

5 - 6 pills

$>=7$ pills

Travel time to the clinic during hypertension visits (in minutes), median (IQR)

$14.0(9.8-29.0)$

$*_{n}=147$; Note: Characteristics are taken at baseline QUICK-I. Age is newly calculated using the date of QUICK-II baseline assessment and the date of birth recorded in QUICK-I; **Based on end-line QUICK-I assessment; ***n = 140. 
Table 2. Changes in adherence to medications and behavioral advice, BP and BMI between baseline and endline.

\begin{tabular}{|c|c|c|c|}
\hline Measures & Baseline $(\mathrm{N}=140)$ & Endline $(N=140)$ & $\begin{array}{c}\mathrm{p} \text {-value } \\
\text { (baseline vs. end line) }\end{array}$ \\
\hline Adherence to medication (Morisky), $\mathrm{n}(\%)$ & & & $<0.001$ \\
\hline Low adherence $(<6)$ & $31(22.1)$ & $16(11.4)$ & \\
\hline Medium adherence (6 - 7.9) & 39 (27.9) & $23(16.4)$ & \\
\hline High adherence (=8) & $70(50.0)$ & $101(72.1)$ & \\
\hline Adherent to behavioral advices, $\mathrm{n}(\%)$ & $106(75.7)$ & $126(90.0)$ & $<0.001$ \\
\hline Alcohol use, n (\%) & $8(5.7)$ & $3(2.1)$ & 0.063 \\
\hline Tobacco use, n (\%) & $6(4.3)$ & $1(0.7)$ & 0.125 \\
\hline Moderate physical activity, n (\%) & $98(70.0)$ & $124(89.2)^{*}$ & $<0.001$ \\
\hline Salt use, n (\%) & $72(51.8)^{*}$ & $72(51.4)$ & 1.000 \\
\hline Fully adherent to medication and behavioral advice, $\mathrm{n}(\%)$ & $58(41.4)$ & $94(67.1)$ & $<0.001$ \\
\hline Systolic BP, median (IQR) & $129.0(118.3-147.3)$ & $122.0(108.0-138.0)$ & $<0.001$ \\
\hline Diastolic BP, median (IQR) & $80.0(71.3-87.8)$ & $73.5(65.0-85.0)$ & $<0.001$ \\
\hline Hypertension classification (JNC7), n (\%) & & & $<0.001$ \\
\hline Normal (SBP < 120 and DBP < 80) & $34(24.3)$ & $65(46.4)$ & \\
\hline Pre-hypertension (SBP 120 - 139 or DBP 80 - 89) & $57(40.7)$ & $40(28.6)$ & \\
\hline Stage 1 hypertension (SBP 140 - 159 or DBP 90 - 99) & $30(21.4)$ & $24(17.1)$ & \\
\hline Stage 2 hypertension (SBP $\geq 160$ or $\mathrm{DBP} \geq 100$ ) & 19 (13.6) & $11(7.9)$ & \\
\hline Body Mass Index, median (IQR) & $25.1(22.2-29.3)$ & $24.9(22.2-29.4)$ & 0.444 \\
\hline Body Mass Index, n (\%) & & & 0.739 \\
\hline Normal weight $(<25)$ & 69 (49.3) & $71(50.7)$ & \\
\hline Overweight (25 - 29.9) & $43(30.7)$ & $40(28.6)$ & \\
\hline Obesity $(\geq 30)$ & $28(20.0)$ & $29(20.7)$ & \\
\hline
\end{tabular}

BP, blood pressure; SBP, systolic blood pressure; DBP, diastolic blood pressure. ${ }^{*} \mathrm{n}=139$.

who reported to always adhere to behavioral advice increased from $106(76 \%)$ at $\mathrm{T}_{0}$ to $126(90 \%)$ at $\mathrm{T}_{1}$ ( $\mathrm{p}<$ 0.001). Median SBP and DBP levels dropped significantly from $129.0 \mathrm{mmHg}$ (IQR: 118.3 - 147.3) to 122.0 mmHg (IQR: 108.0 - 138.0) (p < 0.001) and from $80.0 \mathrm{mmHg}$ (IQR: 71.3 - 87.8) to $73.5 \mathrm{mmHg}$ (IQR: 65.0 85.0) $(\mathrm{p}<0.001)$, respectively. No significant change was found for BMI $(\mathrm{p}=0.444)$. However, more participants reported engaging in moderate physical activity for 30 or more minutes a day, on 3 or more days a week at $\mathrm{T}_{1}: 124(89 \%)$ versus $98(70 \%)$ at $\mathrm{T}_{0}(\mathrm{p}<0.001)$. No statistically significant changes were observed in dietary salt intake, smoking or alcohol consumption between $\mathrm{T}_{0}$ and $\mathrm{T}_{1}$.

\subsection{Improvement in Treatment Adherence Associated with Improvement in BP}

Although trends were positive, no statistically significant associations was observed between BP improvement and changes in medication adherence $(\mathrm{OR}=1.55, \mathrm{p}=0.351)$ and adherence to behavioral recommendations $(\mathrm{OR}=1.93, \mathrm{p}=0.327)$ during the study period (Table 3$)$.

But when using our second definition of BP improvement (Table 4), improvement in medication adherence during the study was associated with a $9.2 \mathrm{mmHg}(\mathrm{p}=0.038)$ reduction in SBP and a $6.1 \mathrm{mmHg}(\mathrm{p}=0.027)$ reduction in DBP. The improvement in adherence to behavioral recommendations was not associated with decrease in BP (SBP: $p=0.214$; DBP: $p=0.318$ ). Included in the definition of improvements in medication adhe- 
Table 3. Association between treatment adherence and improvement in BP at six months; multivariable logistic regression models.

\begin{tabular}{|c|c|c|c|}
\hline Measures & $\begin{array}{l}\text { Improvement in BP*, } \mathrm{n}(\%) \\
(\mathrm{N}=140)\end{array}$ & OR (95\% CI) & p-value \\
\hline \multicolumn{4}{|l|}{ Medication adherence ${ }^{* *}$} \\
\hline Ref: Did not improve at six months & $21(15.0)$ & 1.00 & \\
\hline Improved at six months & $87(62.1)$ & $1.55(0.62-3.86)$ & 0.351 \\
\hline \multicolumn{4}{|l|}{ Behavioral adherence } \\
\hline Ref: Did not improve at six months & $7(5.0)$ & 1.00 & \\
\hline Improved at six months & $101(72.1)$ & $1.93(0.52-7.15)$ & 0.327 \\
\hline
\end{tabular}

*Improvement in blood pressure (BP) is defined as having BP on target at endline or a > 10\% decline in BP at endline compared to baseline; **Improvement in adherence to medication/behavioral advice is defined as having moved to a higher category of adherence between $\mathrm{T}_{0}$ and $\mathrm{T}_{1}$, or as having remained in the highest category of adherence at both time points.

Table 4. Association between treatment adherence and improvement in BP at six months; multivariable linear regression model.

\begin{tabular}{clc}
\hline Measures & \multicolumn{1}{c}{ All respondents $(\mathrm{N}=140)$} & p-value \\
\hline 1. Systolic BP change & Coef. $(95 \% \mathrm{CI})$ & 0.038 \\
Improved medication adherence* & $-9.2(-17.9$ to -0.5$)$ & 0.214 \\
Improved behavioral adherence & $-8.4(-21.6$ to 4.9$)$ & 0.027 \\
2. Diastolic BP change & $-6.1(-11.5$ to -0.7$)$ & 0.318 \\
\hline Improved medication adherence & $-4.2(-12.4$ to 4.1$)$ & \\
\hline Improved behavioral adherence & & \\
\hline
\end{tabular}

*Improved adherence to medication/behavioral advice is defined as having moved to a higher category of adherence between $\mathrm{T}_{0}$ and $\mathrm{T}_{1}$, or as having remained in the highest category of adherence at both time points.

rence are the patients that remained adherent at $T_{0}$ and $T_{1}$. In this subgroup we observed no association between medication adherence and decrease in SBP $(p=0.610)$ and DBP $(p=0.820)$ (results not shown).

\subsection{Illness Perceptions, Medication Beliefs and Self-Efficacy and Medication Adherence}

As intended by CHEP, statistically significant differences between $\mathrm{T}_{0}$ and $\mathrm{T}_{1}$ were observed in some of the illness perception variables, namely for the dimensions timeline chronic $(\mathrm{p}<0.001)$, consequences $(\mathrm{p}<0.001)$, timeline cyclical $(p=0.001)$ and emotional representations $(p<0.001)$ (Table 5). Similarly, statistically significant changes between $T_{0}$ and $T_{1}$ were observed with respect to participants' medication self-efficacy $(\mathrm{p}<0.001)$, beliefs about the necessity of medications ( $\mathrm{p}<0.001$ ), and their concerns about adverse effects of medications ( $\mathrm{p}$ $=0.002)$ (Table 5).

An improvement in medication adherence during the study period was associated with an increase in medication self-efficacy $(\mathrm{OR}=5.99, \mathrm{p}<0.001)$ and a decrease in patients' concerns about adverse effects of medications $(\mathrm{OR}=2.57, \mathrm{p}=0.045)$ (Table 6).

The association between improved adherence to behavioral advice and illness perceptions, medication beliefs and self-efficacy could not be assessed because of the high number of participants who reported a high adherence to behavioral advice at $\mathrm{T}_{0}(76 \%)$ and $\mathrm{T}_{1}(89 \%)$, (Table 2$)$.

Furthermore, in the multivariate analysis, the most important determinants of the improvements in medication adherence (directly), and BP control (indirectly) were changes in perceived medication self-efficacy (OR = 5.99, $\mathrm{p}<0.001)$; concerns about medications $(\mathrm{OR}=2.57, \mathrm{p}=0.045)$; and personal control $(\mathrm{OR}=0.45, \mathrm{p}=0.092)$, although the latter association was not statistically significant. 
Table 5. Changes in illness perceptions, medication self-efficacy and beliefs about medicines between baseline and endline.

\begin{tabular}{ccccc}
\hline Measures & $\begin{array}{c}\text { Baseline }(\mathrm{N}=140) \\
\text { median (IQR) }\end{array}$ & $\begin{array}{c}\text { Endline }(\mathrm{N}=140) \\
\text { median (IQR) }\end{array}$ & $\begin{array}{c}\text { p-value } \\
\text { (baseline vs. end line) }\end{array}$ & Min-max \\
\hline Illness perceptions (IPQ) & $2.6(2.3-3.0)$ & $3.0(2.5-4.0)$ & $<0.001$ & $(1.0-5.0)$ \\
Timeline chronic & $2 .(1.4-3.0)$ & $1.4(1.0-2.2)$ & $<0.001$ & $(1.0-5.0)$ \\
Consequences & $2.0(1.7-2.7)$ & $2.0(1.7-3.0)$ & 0.166 & $(1.0-5.0)$ \\
Personal control & $4.3(4.0-4.7)$ & $4.7(4.0-5.0)$ & 0.080 & $(1.0-5.0)$ \\
Treatment control & $3.0(1.0-4.0) *$ & $3.0(1.0-4.0)$ & 0.179 & $(1.0-5.0)$ \\
Illness coherence & $3.0(3.0-3.5)$ & $3.5(3.0-4.5)$ & 0.001 & $(1.0-5.0)$ \\
Timeline cyclical & $2.5(1.8-3.6)$ & $1.8(1.0-3.3)$ & $<0.001$ & $(1.0-5.0)$ \\
Emotional representations & $3.8(3.4-4.0)$ & $4.0(3.8-4.0)$ & $<0.001$ & $(1.0-4.0)$ \\
Self-efficacy medication (MASES-R) & & & & $(5.0-25.0)$ \\
Beliefs about medication (BMQ) & $20.0(17.0-23.0)$ & $23.0(20.0-25.0)$ & $<0.001$ & $(5.0-25.0)$ \\
Necessity & $10.0(9.0-14.0)$ & $9.0(7.0-13.0)$ & 0.002 & \\
Concern & & &
\end{tabular}

$* \mathrm{n}=139$.

Table 6. Associations between changes in IPQ, MASES-R, BMQ, and improvement in medication adherence; multivariable logistic regression models.

Improvement in medication$$
\text { adherence, } \mathrm{n}(\%)
$$

$(\mathrm{N}=140)$

OR

$95 \%$ CI

p-value

Illness perceptions (IPQ)

\section{Consequences}

Ref: decrease + no change (low + med)

Increase + no change (high)

Personal control

Ref: decrease + no change (low + med)

Increase + no change (high)

$59(42.1)$

$51(36.4)$

$31(22.1)$

1.00

79 (56.4)

13 (9.3)

97 (69.3)

1.00

0.83

$(0.29-2.38)$

0.728

Emotional representations

Decrease + no change (low)

Self-efficacy medication (MASES-R)

Ref: decrease + no change (low + med)

Increase + no change (high)
Beliefs about medicine (BMQ)

\section{Concern}

Ref: Increase + no change (med + high)

Decrease + no change (low)
$37(26.4) \quad 1.00$

$73(52.1) \quad 2.57$

$(1.02-6.51)$

0.045 


\section{Discussion}

Our study demonstrated that a tailored group-based cardiovascular health education program strengthened guideline-based CVD prevention care among hypertensive patients from primary care clinic in a rural community in Nigeria. The patients in question did not adhere to treatment recommendations or had a blood pressure outside the normal range after they had received guideline-based care alone for one year. We observed that $89 \%$ of the patients completed all educational sessions. Studies of similar educational interventions in primary care settings in Europe and the USA recorded lower attendance rates, namely 79\% and 58\% [23] [41]. The high attendance rate in this study suggests that CHEP responded to patients' needs, which is plausible in the light of a previous qualitative study that was conducted in the area [29].

Secondly, we observed that patients who attended CHEP showed improvements in adherence to medication and behavioral recommendations. These improvements may, of course, simply be explained by the fact that patients knew that their hypertension management and adherence was monitored in this study (Hawthorn effect). However, our study provides several indications that the intervention itself has contributed to the improvement in medication adherence. The education program was specifically designed to address previously identified contextual or behavioral barriers to treatment adherence, including patients' perceptions about hypertension and the treatment (see Additional File 1). We found that improved medication adherence after CHEP was positively associated with improved medication self-efficacy (MASES-R) and with a reduction of concerns about medications as measured by the BMQ. It is unlikely that changes in these underlying determinants of adherence to hypertension treatment [42]-[49] would have occurred without the educational intervention. More patients had begun to engage in moderate physical activity during the study period. This improvement may be explained by the fact that CHEP addressed cultural barriers to physical exercise in the community and suggested opportunities for exercise that are part of people's usual everyday activities, such as yam pounding, drawing water from the well, walking, dancing, clapping, fishing or farming. However, for salt use and other behavioral risk factors for CVD no improvements were observed despite the fact that possibilities for changing these behaviors were also specifically addressed during CHEP. Anthropological studies have indicated that dietary practices are particularly difficult to change as they are an important component of one's culture and cultural identity [50]. Changes in dietary behaviour may need more specific approaches than CHEP could offer in three sessions [51]-[53].

Third, we did not find the expected $>10 \%$ decline in SBP/DBD. However, we observed that the median SBP declined with $9.2 \mathrm{mmHg}(\mathrm{p}<0.001)$ and the median DBP with $6.1 \mathrm{mmHg}(\mathrm{p}<0.001)$. Moreover, after the study period, more patients could be classified as having a BP within the normal range according to the JNC7 hypertension classification system. These results are, as such, clinically relevant [54]. Moreover, we found that the decline of the median BP levels was associated with an improvement in medication adherence.

To our knowledge this is one of the first studies that analyzed the potential impact of hypertension education in the context of a CBHI program that aims to improve the quality of CVD prevention in low resource primary care setting in Africa. The study is also unique in its explicit description of the educational intervention and its potential replicability by healthcare providers and researchers in other settings (Additional File 1).

\subsection{Limitations}

Yet, this study has several limitations, the most important being the lack of a control group. Although, we observed significant improvements in outcome measures, the lack of a control group limits the possibility of drawing firm conclusions as to the causality of the measured effects. The link between CHEP and behavioral and clinical outcomes should be further tested in randomized controlled trials or prospective studies. Medication adherence (MMAS-8) and behavioral adherence were measured through self-report scales, and answers could have been influenced by social desirability. Nevertheless, MMAS-8 is a validated, reliable, simple, and low-cost instrument that has been successfully used to estimate medication adherence in many previous studies involving hypertensive patients [55]-[59], including low-income patients of African origin [36]. Furthermore, due to the relatively small sample size, only a limited number of variables could be taken into consideration within our multivariate analysis. We have opted for the inclusion of variables that refer to behavioral determinants of adherence. The inclusion of additional variables such as the type or the number of medications used might have strengthened this study. In addition, the limited sample size also made it impossible to conduct sub-group analyses, for instance for patients with different levels of formal school education or for those with different levels of treatment adherence at the start of the study. Furthermore, to evaluate the long-term effect of CHEP longer follow-up studies are needed. Finally, 
a recent study from Nigeria reported that the adherence level was higher among hypertensive patients attending specialized clinics compared to those attending general outpatient clinics, despite the former's use of more medications [60]. In future studies evaluating CHEP, attention should be given to the influence of the context in which care is provided and to the type and the number of medications patients are being prescribed.

CHEP was designed to meet the specific needs of the study population. Some of the culturally specific issues that were addressed by CHEP may not be relevant to patients who live in other socio-cultural settings. Yet the description of CHEP provides general outlines for structuring and providing patient education, which makes it possible to adapt the specific contents to the needs of other patient populations. It should be realized, however, that the patients in our study had access to free primary care through health insurance. The findings might therefore not automatically be generalizable to the broader group of (mostly uninsured) hypertensive patients in the larger hypertensive population in Africa or to those who are treated at secondary and tertiary levels of care. Finally, the study was conducted in a health care facility that had participated in a CVD quality improvement program (for over a year) that was subsidized by a CBHI program. It is likely that the usual care that was being provided in this facility is better than that in other facilities. Consequently, the effects of CHEP might have been greater if we had conducted the study in a common primary care setting in Nigeria or elsewhere in Africa.

\subsection{Implication for Practice and Further Research}

This study found that CHEP responds to patients' needs and that it can be a useful component of the primary care management of hypertension in low resource communities in SSA, if it is combined with appropriate pharmaceutical treatment. Further (controlled) studies are needed to confirm or refute these findings. This type of health education can be delivered (efficiently) by nurses and other trained health workers, and not just by physicians. The modules of the cardiovascular health education program (CHEP) are well described (Additional File 1) and can serve as a (useful) framework for further development and evaluation of educational interventions for patients who are at risk of developing CVD, and particularly for those living in disadvantaged communities in SSA.

\section{Conclusion}

This study suggested that the evaluated education program (CHEP) improved adherence to medications, followed by an increase in BP control among insured hypertensive patients in rural Nigeria. At the end of the study, more participants reported high adherence to medications and behavioral advice than at baseline: respectively, $101(72 \%)$ versus 70 (50\%), ( $<$ < 0.001) and $126(90 \%)$ versus $106(76 \%)$, $(\mathrm{p}<0.001)$. Participants with controlled BP doubled from $34(24 \%)$ to $65(46 \%),(\mathrm{p}=0.001)$. The median SBP and DBP decreased from 129.0 to $122.0 \mathrm{mmHg}$, ( $=0.002)$ and from 80.0 to $73.5 \mathrm{mmHg}$, $(\mathrm{p}<0.001)$, respectively. BMI did not change $(\mathrm{p}=$ $0.444)$. Improved medication adherence was associated with a decrease in medication concerns $(p=0.045)$ and improved medication self-efficacy ( $\mathrm{p}<0.001$ ). Making such programs available to affected populations in SSA has the potential to help reduce burden of cardiovascular diseases and associated mortality.

\section{Competing Interests}

A.O. Odusola received a grant (\# CF7536/2011) from NUFFIC. The study was funded by Health Insurance Fund. The Fund was not involved in the study design, data collection, analysis, and interpretation or reporting of the data. The remaining authors declare that they have no competing interests.

\section{Author Contributions}

AOO drafted the manuscript, conducted the study and participated in the design, reporting, analysis and revision. $\mathrm{JH}, \mathrm{JL}, \mathrm{KS}$ and MH participated in the original study design. JH and KS made substantial revision of several drafts of the manuscript. HN conducted the statistical analyses with critical contributions from FW. AOO drafted the education program with critical contributions from JH. AO, CA, CS, FW, GO, HN, JH, KS, MH, $\mathrm{OAB}$ and TA reviewed the manuscript critically. KA and PA provided vital logistic supports. CS, JH and KS reviewed the data collection and management procedures. AO, TA, CS, JH, and KS are members of the supervisory board. All authors read and approved the final draft. 


\section{Acknowledgements}

Special tributes go to late Prof. Joep Lange, who tragically passed away before the manuscript had been finished. He initiated and promoted an enabling environment for the study and related projects. We thank the Medical Director and staff of Ogo Oluwa hospital for facilitating a successful data collection. We are grateful to all participants for collaboration in the study. We thank HIF, Pharm Access Foundation, KSHI and NUFFIC for supporting the study.

\section{Additional Files}

Additional File 1: (1) CSV; Table; Cardiovascular Health Education Program (CHEP); Overview of groupbased Cardiovascular Health Education Program used in QUICK-II study; (2) CSV; Table; Changes in inclusion characteristics of QUICK-II participants between endline QUICK-I and baseline QUICK-II assessments.

\section{References}

[1] WHO (2011) Global Atlas on Cardiovascular Disease Prevention and Control. http://www.who.int/cardiovascular_diseases/publications/atlas_cvd/en/

[2] Alwan, A. and WHO (2011) Global Status Report on Non-Communicable Diseases 2010. http://whqlibdoc.who.int/publications/2011/9789240

[3] WHO and Others (2007) Prevention of Cardiovascular Disease: Pocket Guidelines for Assessment and Management of Cardiovascular Risk (WHO/ISH Cardiovascular Risk Prediction Charts for the European Region).

[4] Mendis, S., Lindholm, L.H., Mancia, G., Whitworth, J., Alderman, M., Lim, S., et al. (2007) World Health Organization (WHO) and International Society of Hypertension (ISH) Risk Prediction Charts: Assessment of Cardiovascular Risk for Prevention and Control of Cardiovascular Disease in Low and Middle-Income Countries. Journal of Hypertension, 25, 1578-1582. http://dx.doi.org/10.1097/HJH.0b013e3282861fd3

[5] Lewington, S., Clarke, R., Qizilbash, N., Peto, R. and Collins, R. (2002) Prospective Studies Collaboration: Age-Specific Relevance of Usual Blood Pressure to Vascular Mortality: A Meta-Analysis of Individual Data for One Million Adults in 61 Prospective Studies. Lancet, 360, 1903-1913. http://dx.doi.org/10.1016/S0140-6736(02)11911-8

[6] Kayima, J., Wanyenze, R.K., Katamba, A., Leontsini, E. and Nuwaha, F. (2013) Hypertension Awareness, Treatment and Control in Africa: A Systematic Review. BMC Cardiovascular Disorders, 13, 1-11. http://dx.doi.org/10.1186/1471-2261-13-54

[7] Ogah, O.S., Okpechi, I., Chukwuonye, I.I., Akinyemi, J.O., Onwubere, B.J., Falase, A.O., et al. (2012) Blood Pressure, Prevalence of Hypertension and Hypertension Related Complications in Nigerian Africans: A Review. World Journal of Cardiology, 4, 327-340. http://dx.doi.org/10.4330/wjc.v4.i12.327

[8] Beaglehole, R., Epping-Jordan, J., Patel, V., Chopra, M., Ebrahim, S., Kidd, M., et al. (2008) Improving the Prevention and Management of Chronic Disease in Low-Income and Middle-Income Countries: A Priority for Primary Health Care. The Lancet, 372, 940-949. http://dx.doi.org/10.1016/S0140-6736(08)61404-X

[9] Mendis, S., Abegunde, D., Oladapo, O., Celletti, F. and Nordet, P. (2004) Barriers to Management of Cardiovascular Risk in a Low-Resource Setting Using Hypertension as an Entry Point. Journal of Hypertension, 22, 59-64. http://dx.doi.org/10.1097/00004872-200401000-00013

[10] Maimaris, W., Paty, J., Perel, P., Legido-Quigley, H., Balabanova, D., Nieuwlaat, R., et al. (2013) The Influence of Health Systems on Hypertension Awareness, Treatment, and Control: A Systematic Literature Review. PLoS Medicine, 10, e1001490. http://dx.doi.org/10.1371/journal.pmed.1001490

[11] Adebisi, O.O. and Samali, A. (2013) Poverty and Hypertension in Nigerian Adults: A Barrier to Its Control and Treatment: A Review. Unique Research Journals, 1, 013-014.

[12] Mwaura, J.W. and Pongpanich, S. (2012) Access to Health Care: The Role of a Community Based Health Insurance in Kenya. The Pan African Medical Journal, 12, 35.

[13] Onoka, C., Onwujekwe, O., Uzochukwu, B. and Ezumah, N. (2013) Promoting Universal Financial Protection: Constraints and Enabling Factors in Scaling-Up Coverage with Social Health Insurance in Nigeria. Health Research Policy and Systems, 11, 20. http://dx.doi.org/10.1186/1478-4505-11-20

[14] Hendriks, M.E., Wit, F.W., Akande, T.M., Kramer, B., Osagbemi, G.K., Tanović, Z., et al. (2014) Effect of Health Insurance and Facility Quality Improvement on Blood Pressure in Adults With Hypertension in Nigeria A Population-Based Study. JAMA Internal Medicine, 174, 555-563. http://dx.doi.org/10.1001/jamainternmed.2013.14458

[15] Krousel-Wood, M., Thomas, S., Muntner, P. and Morisky, D. (2004) Medication Adherence: A Key Factor in Achiev- 
ing Blood Pressure Control and Good Clinical Outcomes in Hypertensive Patients. Current Opinion in Cardiology, 19, 357-362. http://dx.doi.org/10.1097/01.hco.0000126978.03828.9e

[16] Bowry, A.D., Shrank, W.H., Lee, J.L., Stedman, M. and Choudhry, N.K. (2011) A Systematic Review of Adherence to Cardiovascular Medications in Resource-Limited Settings. Journal of General Internal Medicine, 26, 1479-1491. http://dx.doi.org/10.1007/s11606-011-1825-3

[17] AlGurair, S., Hughes, C., Simpson, S. and Guirguis, L. (2012) A Systematic Review of Patient Self-Reported Barriers of Adherence to Antihypertensive Medication Using the World Health Organization Multidimensional Adherence Model. The Journal of Clinical Hypertension, 14, 877-886. http://dx.doi.org/10.1111/j.1751-7176.2012.00699.x

[18] De Geest, S. and Sabate, E. (2003) Adherence to Long-Term Therapies: Evidence for Action. European Journal of Cardiovascular Nursing, 2, 323. http://dx.doi.org/10.1016/S1474-5151(03)00091-4

[19] Kleinman, A., Eisenberg, L. and Good, B. (2006) Culture, Illness, and Care: Clinical Lessons from Anthropologic and Cross-Cultural Research. FOCS, 4, 140-149. http://dx.doi.org/10.1176/foc.4.1.140

[20] Leventhal, H., Brissette, I. and Leventhal, E.A. (2003) The Common-Sense Model of Self-Regulation of Health and Illness. The Self-Regulation of Health and Illness Behaviour, 1, 42-65.

[21] Marshall, I.J., Wolfe, C.D. and McKevitt, C. (2012) Lay Perspectives on Hypertension and Drug Adherence: Systematic Review of Qualitative Research. BMJ, 345, e3953-e3953. http://dx.doi.org/10.1136/bmj.e3953

[22] Houston, T.K., Allison, J.J., Sussman, M., Horn, W., Holt, C.L., Trobaugh, J., et al. (2011) Culturally Appropriate Storytelling to Improve Blood Pressure: A Randomized Trial. Annals of Internal Medicine, 154, 77-84. http://dx.doi.org/10.7326/0003-4819-154-2-201101180-00004

[23] Beune, E.J., van Charante, E.P.M., Beem, L., Mohrs, J., Agyemang, C.O., Ogedegbe, G., et al. (2014) Culturally Adapted Hypertension Education (CAHE) to Improve Blood Pressure Control and Treatment Adherence in Patients of African Origin with Uncontrolled Hypertension: Cluster-Randomized Trial. PloS ONE, 9, e90103. http://dx.doi.org/10.1371/journal.pone.0090103

[24] González-fernández, R.A., Rivera, M., Torres, D., Quiles, J. and Jackson, A. (1990) Usefulness of a Systemic Hypertension In-Hospital Educational Program. American Journal of Cardiology, 65, 1384-1386. http://dx.doi.org/10.1016/0002-9149(90)91332-Z

[25] Warsi, A., Wang, P.S., LaValley, M.P., Avorn, J. and Solomon, D.H. (2004) Self-management Education Programs in Chronic Disease: A Systematic Review and Methodological Critique of the Literature. Archives of Internal Medicine, 164, 1641-1649. http://dx.doi.org/10.1001/archinte.164.15.1641

[26] Walsh, J.M., Sundaram, V., McDonald, K., Owens, D.K. and Goldstein, M.K. (2008) Implementing Effective Hypertension Quality Improvement Strategies: Barriers and Potential Solutions. The Journal of Clinical Hypertension, 10, 311-316. http://dx.doi.org/10.1111/j.1751-7176.2008.07425.x

[27] Mendis, S., Johnston, S.C., Fan, W., Oladapo, O., Cameron, A. and Faramawi, M.F. (2010) Cardiovascular Risk Management and Its Impact on Hypertension Control in Primary Care in Low-Resource Settings: A Cluster-Randomized Trial. Bulletin of the World Health Organization, 88, 412-419. http://dx.doi.org/10.2471/BLT.08.062364

[28] Hendriks, M.E., Bolarinwa, O.A., Wit, F.W., Brewster, L.M., Odusola, A.O., Rosendaal, N.T., et al. (2014) Feasibility and Quality of Cardiovascular Disease Prevention within a Community-Based Health Insurance Program in Rural Nigeria: An Operational Cohort Study. Journal of Hypertension, 33, 366-375.

[29] Odusola, A.O., Hendriks, M., Schultsz, C., Bolarinwa, O.A., Akande, T., Osibogun, A., et al. (2014) Perceptions of Inhibitors and Facilitators for Adhering to Hypertension Treatment among Insured Patients in Rural Nigeria: A Qualitative Study. BMC Health Services Research, 14, 624. http://dx.doi.org/10.1186/s12913-014-0624-z

[30] Odusola, A.O., Hendriks, M., Schultsz, C., Stronks, K., Lange, J., Osibogun, A., et al. (2011) Development and Evaluation of a Patient Centered Cardiovascular Health Education Program for Insured Patients in Rural Nigeria (QUICK-II). BMC Public Health, 11, 171. http://dx.doi.org/10.1186/1471-2458-11-171

[31] Health Insurance Fund. http://www.hifund.org/

[32] American Diabetes Association (2010) Standards of Medical Care in Diabetes—2010. Diabetes Care, 33, S11-S61. http://dx.doi.org/10.2337/dc10-S011

[33] Mancia, G., Fagard, R., Narkiewicz, K., Redon, J., Zanchetti, A., Böhm, M., et al. (2013) 2013 ESH/ESC Guidelines for the Management of Arterial Hypertension. The Task Force for the Management of Arterial Hypertension of the European Society of Hypertension (ESH) and of the European Society of Cardiology (ESC). European Heart Journal, 34, 2159-2219. http://dx.doi.org/10.1093/eurheartj/eht151

[34] Hendriks, M., Brewster, L., Wit, F., Bolarinwa, O.A., Odusola, A.O., Redekop, W., et al. (2011) Cardiovascular Disease Prevention in Rural Nigeria in the Context of a Community Based Health Insurance Scheme: Quality Improvement Cardiovascular Care Kwara-I (QUICK-I). BMC Public Health, 11, 186. http://dx.doi.org/10.1186/1471-2458-11-186 
[35] Morisky, D.E., Green, L.W. and Levine, D.M. (1986) Concurrent and Predictive Validity of a Self-Reported Measure of Medication Adherence. Medical Care, 24, 67-74. http://dx.doi.org/10.1097/00005650-198601000-00007

[36] Morisky, D.E., Ang, A., Krousel-Wood, M. and Ward, H.J. (2008) Predictive Validity of a Medication Adherence Measure in an Outpatient Setting. The Journal of Clinical Hypertension, 10, 348-354. http://dx.doi.org/10.1111/j.1751-7176.2008.07572.x

[37] Moss-Morris, R., Weinman, J., Petrie, K., Horne, R., Cameron, L. and Buick, D. (2002) The Revised Illness Perception Questionnaire (IPQ-R). Psychology \& Health, 17, 1-16. http://dx.doi.org/10.1080/08870440290001494

[38] Horne, R., Weinman, J. and Hankins, M. (1999) The Beliefs about Medicines Questionnaire: The Development and Evaluation of a New Method for Assessing the Cognitive Representation of Medication. Psychology \& Health, 14, 124. http://dx.doi.org/10.1080/08870449908407311

[39] Ogedegbe, G., Mancuso, C.A., Allegrante, J.P. and Charlson, M.E. (2003) Development and Evaluation of a Medication Adherence Self-Efficacy Scale in Hypertensive African-American Patients. Journal of Clinical Epidemiology, 56, 520-529. http://dx.doi.org/10.1016/S0895-4356(03)00053-2

[40] Fernandez, S., Chaplin, W., Schoenthaler, A. and Ogedegbe, G. (2008) Revision and Validation of the Medication Adherence Self-Efficacy Scale (MASES) in Hypertensive African Americans. Journal of Behavioral Medicine, 31, 453-462. http://dx.doi.org/10.1007/s10865-008-9170-7

[41] Ogedegbe, G., Tobin, J.N., Fernandez, S., Cassells, A., Diaz-Gloster, M., Khalida, C., et al. (2014) Counseling African Americans to Control Hypertension: Cluster-Randomized Clinical Trial Main Effects. Circulation, 129, $2044-2051$. http://dx.doi.org/10.1161/CIRCULATIONAHA.113.006650

[42] Sweileh, W.M., Zyoud, S.H., Abu Nab’a, R.J., Deleq, M.I., Enaia, M.I., Nassar, S.M., et al. (2014) Influence of Patients' Disease Knowledge and Beliefs about Medicines on Medication Adherence: Findings from a Cross-Sectional Survey among Patients with Type 2 Diabetes Mellitus in Palestine. BMC Public Health, 14, 94. http://dx.doi.org/10.1186/1471-2458-14-94

[43] AlHewiti, A. (2014) Adherence to Long-Term Therapies and Beliefs about Medications. International Journal of Family Medicine, 2014, Article ID: 479596.

[44] Ruppar, T.M., Dobbels, F. and De Geest, S. (2012) Medication Beliefs and Antihypertensive Adherence among Older Adults: A Pilot Study. Geriatric Nursing, 33, 89-95. http://dx.doi.org/10.1016/j.gerinurse.2012.01.006

[45] Schoenthaler, A., Ogedegbe, G. and Allegrante, J.P. (2009) Self-Efficacy Mediates the Relationship Between Depressive Symptoms and Medication Adherence Among Hypertensive African Americans. Health Education \& Behavior, 36, 127-137. http://dx.doi.org/10.1177/1090198107309459

[46] Gatti, M.E., Jacobson, K.L., Gazmararian, J.A., Schmotzer, B. and Kripalani, S. (2009) Relationships between Beliefs about Medications and Adherence. American Journal of Health-System Pharmacy, 66, 657-664.

http://dx.doi.org/10.2146/ajhp080064

[47] Chen, S.-L., Tsai, J.-C. and Lee, W.-L. (2009) The Impact of Illness Perception on Adherence to Therapeutic Regimens of Patients with Hypertension in Taiwan. Journal of Clinical Nursing, 18, 2234-2244. http://dx.doi.org/10.1111/j.1365-2702.2008.02706.x

[48] Ross, S., Walker, A. and MacLeod, M.J. (2004) Patient Compliance in Hypertension: Role of Illness Perceptions and Treatment Beliefs. Journal of Human Hypertension, 18, 607-613. http://dx.doi.org/10.1038/sj.jhh.1001721

[49] Rajpura, J.R. and Nayak, R. (2014) Role of Illness Perceptions and Medication Beliefs on Medication Compliance of Elderly Hypertensive Cohorts. Journal of Pharmacy Practice, 27, 19-24. http://dx.doi.org/10.1177/0897190013493806

[50] Helman, C.G. (2014) Culture, Health and Illness: An Introduction for Health Professionals. Butterworth-Heinemann, Boston.

[51] Campbell, M.K., DeVellis, B.M., Strecher, V.J., Ammerman, A.S., DeVellis, R.F. and Sandler, R.S. (1994) Improving Dietary Behavior: The Effectiveness of Tailored Messages in Primary Care Settings. American Journal of Public Health, 84, 783-787. http://dx.doi.org/10.2105/AJPH.84.5.783

[52] Campbell, M.K., Honess-Morreale, L., Farrell, D., Carbone, E. and Brasure, M. (1999) A Tailored Multimedia Nutrition Education Pilot Program for Low-Income Women Receiving Food Assistance. Health Education Research, 14, 257-267. http://dx.doi.org/10.1093/her/14.2.257

[53] Abbott, P., Davison, J., Moore, L. and Rubinstein, R. (2010) Barriers and Enhancers to Dietary Behaviour Change for Aboriginal People Attending a Diabetes Cooking Course. Health Promotion Journal of Australia, 21, 33-38.

[54] Staessen, J.A., Wang, J.G. and Thijs, L. (2001) Cardiovascular Protection and Blood Pressure Reduction: A Meta-Analysis. Lancet, 358, 1305-1315. http://dx.doi.org/10.1016/S0140-6736(01)06411-X

[55] Korb-Savoldelli, V., Gillaizeau, F., Pouchot, J., Lenain, E., Postel-Vinay, N., Plouin, P.-F., et al. (2012) Validation of a French Version of the 8-Item Morisky Medication Adherence Scale in Hypertensive Adults. Journal of Clinical Hyper- 
tension, 14, 429-434. http://dx.doi.org/10.1111/j.1751-7176.2012.00634.x

[56] Oliveira-Filho, A.D., Barreto-Filho, J.A., Neves, S.J.F. and Lyra Jr., D.P. (2012) Association between the 8-Item Morisky Medication Adherence Scale (MMAS-8) and Blood Pressure Control. Arquivos Brasileiros De Cardiologia, 99, 649-658. http://dx.doi.org/10.1590/S0066-782X2012005000053

[57] Holt, E., Joyce, C., Dornelles, A., Morisky, D., Webber, L.S., Muntner, P., et al. (2013) Sex Differences in Barriers to Antihypertensive Medication Adherence: Findings from the Cohort Study of Medication Adherence among Older Adults. Journal of the American Geriatrics Society, 61, 558-564. http://dx.doi.org/10.1111/jgs.12171

[58] Lee, G.K.Y., Wang, H.H.X., Liu, K.Q.L., Cheung, Y., Morisky, D.E. and Wong, M.C.S. (2013) Determinants of Medication Adherence to Antihypertensive Medications among a Chinese Population Using Morisky Medication Adherence Scale. PloS ONE, 8, e62775. http://dx.doi.org/10.1371/journal.pone.0062775

[59] Sa'ed, H., Al-Jabi, S.W., Sweileh, W.M. and Morisky, D.E. (2013) Relationship of Treatment Satisfaction to Medication Adherence: Findings from a Cross-Sectional Survey among Hypertensive Patients in Palestine. Health and Quality of Life Outcomes, 11, 191. http://dx.doi.org/10.1186/1477-7525-11-191

[60] Akintunde, A.A. and Akintunde, T.S. (2015) Antihypertensive Medications Adherence among Nigerian Hypertensive Subjects in a Specialist Clinic Compared to a General Outpatient Clinic. Annals of Medical and Health Sciences Research, 5, 173-178. http://dx.doi.org/10.4103/2141-9248.157492 


\section{Additional File 1}

Table 1. Overview of group-based Cardiovascular Health Education Program (CHEP) used in QUICK-II study.

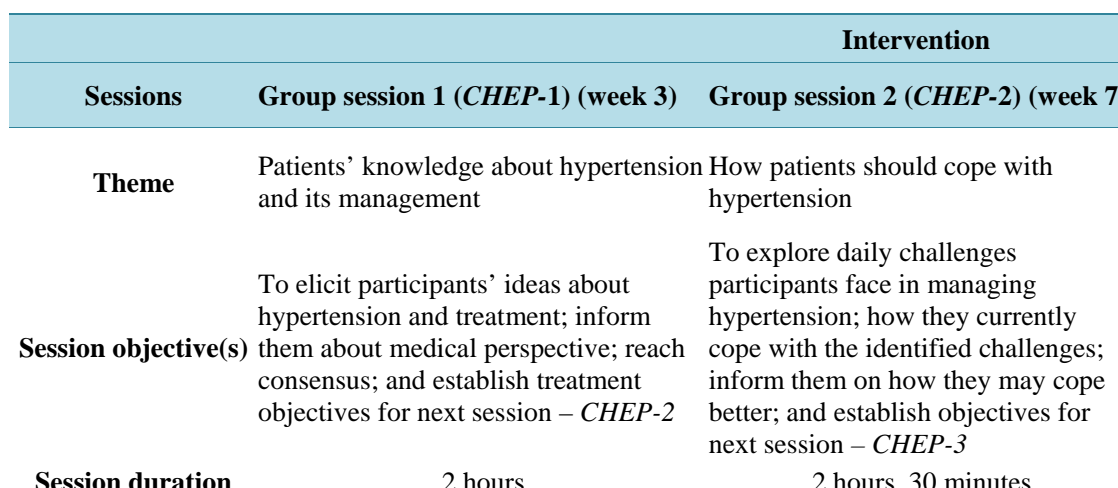

Group session 3 (CHEP-3) (week 15)

How patients should cope with hypertension

To explore daily challenges participants face in managing hypertension; how they currently cope with the identified challenges, inform them on how they may cope better; and establish how they can continue to deal with the challenges in future

$$
2 \text { hours, } 30 \text { minutes }
$$

3.1: Medication use: group discussion (15 minutes)

1.1: Hypertension and its management: group discussion (30 minutes)

Themes:

- What is hypertension?

- Is hypertension a disease?

- What are your views about hypertension?

- What causes hypertension?

- Who can get hypertension?

- Is hypertension dangerous, If so, how dangerous is it?

- Is hypertension curable?

- How long does hypertension last?

- How does hypertension present?

- How did you get your hypertension?

- What can you do to prevent hypertension?

- How can hypertension be treated?

- How is hypertension related to you lifestyle and what you eat?

Protocol 1.2: What patients can do to manage instructions hypertension: interactive instruction (30 minutes)*

Themes:

- Take your medications regularly as prescribed

- $\quad$ Seek support from your family/friends on reminders in taking your drugs regularly and in reducing salt intake

- Adopt healthy diet (described)

- Decrease amount of sodium/salt in your diet

- Stay physically active/exercise regularly

- Lose excess weight

- Quit smoking

- Quit/reduce alcohol intake

- Quit snuff, Kola nuts

- Limit exposure to physical/emotional stress

1.3: Addressing what can make hypertension management difficult for patients: group discussion (30 minutes)
2.1: Medication use: group discussion (15 minutes)

- What challenges do you face?

- How did you cope?

2.2: Dietary advice/salt: group discussion (15 minutes)

- What challenges do you face?

- How did you cope?

2.3: Weight reduction/exercise group discussion (15 minutes)

- What challenges do you face?

- How did you cope?

2.4: Attending your follow-up appointments regularly as advised group discussion (15 minutes)

- What challenges do you face?

- How did you cope?

2.5: Audiovisual 'Living positively with hypertension’: Instruction (25 minutes)

- View and discuss video "living positively with hypertension" and some patient-centred exercise regimes to help coping (35 minutes)

2.6: Simple exercises you can do at home (20 minutes)

- $\quad$ Poster teaching session (5 minutes)

- $\quad$ Practical exercise session (15 minutes)

2.7: Individual assignment (10 minutes):

- $\quad$ Set 3 goals on what you want to achieve before your next CHEP visit to keep your blood pressure controlled (e.g. reduce salt consumption, increase physical activity through exercise)
- What challenges do you face?

- How did you cope?

3.2: Dietary advice/salt: group discussion (15 minutes)

- What challenges do you face?

- How did you cope?

3.3: Weight reduction/exercise group discussion (15 minutes)

- What challenges do you face?

- How did you cope?

3.4: Attending your follow-up appointments regularly as advised group discussion (15 minutes)

- What challenges do you face?

- How did you cope?

3.5: Audiovisual 'Living positively with hypertension': Instruction (25 minutes)

- View and discuss video "living positively with hypertension” and some patient-centered exercise regimes to help coping (35 minutes)

3.6: Simple exercises you can do at home (20 minutes)

- Patient friendly exercise practice sessions using: local activities such as wood cutting, gardening, mortar other household chores, farming, biking, brisk walking, leisure activities e.g. drumming, dancing.

3.7: Final closing session

(Instructions), 10 minutes

In moving forward:

- Make the lessons learnt from this program your daily routine for life

- Take your drugs regularly

- Exercise daily, regularly

- Reduce salt intake and adopt healthy diet

- Attend your follow-up clinic regularly

- Get needed support from family members/friends in your hypertension self-management efforts grinding, drawing water from well, 


\section{Continued}

Themes:

- Taking your pills regularly

- Reducing salt intake

- Adopting a healthy diet

- Staying physically active

1.4: How to overcome obstacles:

instruction (20 minutes) **

1.5: Individual assignment (10

minutes)

- Set 3 goals on what you want to achieve before your next CHEP visit to keep your blood pressure controlled (e.g. reduce salt consumption, increase physical activity through exercise)

\section{Additional information:}

Two instructors guided the sessions

Sessions were held in English, Yoruba and Nupe with a translator

All sessions included a 5 minute welcome

Results of participants' homework assignments were discussed with the trainers 15 minutes before the start of next sessions

During breaks, patients viewed educational posters

*Power points are used; ** Posters are used.

Table 2. Changes in inclusion characteristics of QUICK-II participants between endline QUICK-I and baseline QUICK-II assessments.

\begin{tabular}{ccc}
\hline Measurements & Endline QUICK-I (N = 149) & Baseline QUICK-II (N = 149) \\
\hline High BP no co-morbidities & $36(24.2)$ & $15(10.1)$ \\
High BP with co-morbidities & $17(11.4)$ & $10(6.7)$ \\
Low or medium adherence & $61(40.9)$ & $40(26.9)$ \\
High BP no co-morbidities AND low/med adherence & $31(20.8)$ & $25(16.8)$ \\
High BP with co-morbidities AND low/med adherence & $4(2.7)$ & $10(6.7)$ \\
Low BP and high adherence & $0(0.0)$ & $49(32.9)$ \\
\hline
\end{tabular}

\section{List of Abbreviations}

BMQ: Beliefs about Medicines Questionnaire

BP: Blood pressure

CHEP: Cardiovascular Health Education Program

DBP: Diastolic Blood Pressure

HIF: Health Insurance Fund

IPQ-R: Revised Illness Perception Questionnaire

KSHI: Kwara State Health Insurance

MASES-R: Revised Medication Adherence Self Efficacy Scale

MMAS: Morisky Medication Adherence Scale

NUFFIC: Netherlands organization for international cooperation in higher education

OOH: Ogo Oluwa Hospital

QUICK: Quality Improvement Cardiovascular Care Kwara

SBP: Systolic Blood Pressure 